\title{
Study on electrical energy meter for energy measuring under harmonics condition
}

\author{
Rui Wang ${ }^{\mathrm{a}}$ and Jing Yuan \\ Chongqing Academy of Metrology and Quality Inspection, Chongqing 401123, China
}

\begin{abstract}
In this study, the principle and influence of electrical energy meter measurement were analyzed under harmonics condition. Subsequently, a test platform was set up to investigate the impacts of three typical harmonic waveform on the performance of electrical energy meter. The electric energy measurement results were obtained under sine wave and harmonics conditions. The test results were in accordance with theoretical analyses. It is very useful for the quality of electrical energy meter testing and assessment.
\end{abstract}

Keywords: electrical energy meter; electric energy measurement; harmonics.

\section{Introduction}

Power is considered as an important energy source. Nowadays, it has a significant impact on global economy. Power can be a special commodity; energy meters are used as measuring instruments, therefore their accuracy are very important for the fair trade settlement of power. There are a lot of nonlinear loads appearance in power system, such as frequency converter, metallurgy and chemical industry, electric railway locomotive, which make the inject power grid more complex by increasing harmonic components [1][2]. Furthermore, the nonlinear loads have adversely affects on the electrical measuring instruments (e.g., electric energy meter). Therefore, it is quite necessary to assess the impacts of harmonics conditions on the theoretical and experimental performances of electric energy measurement via electrical energy meter.

\section{Theoretical analysis of electrical energy meter measuring in the influence of harmonics}

The single phase electrical energy meter principle block diagram is shown in Fig. 1. The current and the voltage acted on the solid state (electronic) components, which is proportional to the energy generated in a certain time. Three-phase electrical energy meter, in which each phase voltage, current, and multiplication of the voltage and current were the same of single phase electrical energy meter, while in front of the P/F converter for an additional sum of circuits. The multiplexer output signals were added up to the frequency divider.

a Corresponding author : wanry77@sina.com

(C) 2016. The authors - Published by Atlantis Press 


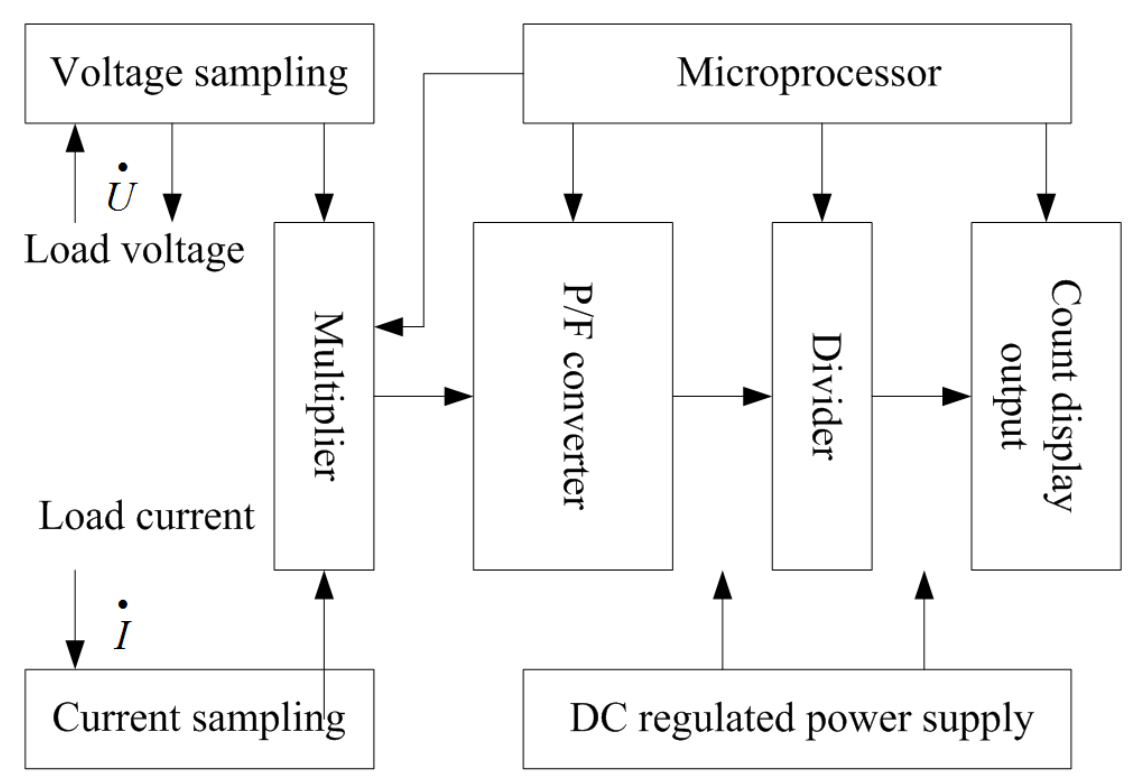

Figure 1. Structure schematic diagram of single-phase electrical energy meter

The frequency characteristics of electrical energy meter were relatively flat. According to the principle of electrical energy meter, the value of electronic energy under harmonics influence during the time $t$ is as follows[3][4]:

$$
W=\left[U_{1} I_{1} \cos \varphi_{1}+\sum U_{n} I_{n} \cos \left(\varphi_{n}+\lambda_{n}\right)\right] T \quad(n=2,3, \cdots)
$$

Where $\lambda_{\mathrm{n}}$ is phase offset of harmonic energy. Because input circuit of electrical energy meter makes the different frequency signals produce different phase offset, phase compensation introduced in $50 \mathrm{~Hz}$ can't compensate the phase offset of other frequency, which brings $\lambda_{\mathrm{n}}$.So measurement basic error change caused by the different frequency change. According to the orthogonality of trigonometric function, when the system voltage and current signal in only one distortion that electrical energy meter measurement error should change, but the error variation would not change significantly. When the current and voltage signals are both distorted that electrical energy meter measurement error should change slightly. Therefore, quality of electrical energy meter is reliable that has a wide frequency characteristic, and can measure accurately the total electrical energy values under the influence of the harmonic.

\section{The test and experimental study of electrical energy meter measuring under influence of harmonics}

To prove the correctness of theory analysis, combining with harmonics impact requirements of the current international standard and international recommendation of electrical energy, the laboratory set up a test platform under the condition of harmonics impacting, test and prove the above conclusion.

\subsection{Test preparation}

The main measurement standards were obtained from the FLUKE 6145A electrical power standard source in the test platform. This standard source can support four-phase system and produce harmonics, simulate harmonic distortion, modulation harmonics, rising and dropping drastically, 
flicker etc.. The voltage output range is $(0 \sim 1008) \mathrm{V}$, and the current output range is (0 21) A. It has (66 PPM)/year accuracy. The output of the voltage and current harmonic can reach 100 times, especially in the calibration options with standard electric energy meter method and watt•hour method. FLUKE 6145A is one of the world's most advanced harmonic power source.

The test samples are chosen representative sample of electrical energy meter manufacturers XX, three phase four wire model for XXX, specifications for 220 (380) V, 1.5 (6) A, the accuracy of 1 class.

The tests were carried out according to requirement of IEC62053-21 Electricity metering equipment (a .c.)-Particular requirements-Part 21 Static meters for active energy (classes 1 and 2) and OIML R46-1/-2 Active electrical energy meters. Part1: Metrological and technical requirements Part2: Metrological controls and performance tests.

\subsection{Test results and analyses}

The tests were conducted in three trials, harmonics in voltage and current, sub-harmonics in the AC current circuit and harmonics in the AC current circuit. The test connection diagram is shown in Fig. 2. The voltage output circuit and current output circuit of the FLUKE 6145A, respectively, connecting to voltage and current input circuit of electric energy meter. The harmonic waveforms needed to test generated by the FLUKE 6145A enter into electric energy meter. Electric energy meter being measured output power pulse into the FLUKE 6145A pulse input terminal. Owing to FLUKE 6145A has function of electric energy measurement, The standard electric energy meter and the pulse comparator were replaced and FLUKE 6145A directly show power measurement error[5].



Figure 2. Schematic diagram of test

\subsubsection{Harmonics in voltage and current}

The test of harmonics in voltage and current is to verify that the error shift due to influence of harmonics in voltage and current. The error shift, compared to the intrinsic error at sinusoidal conditions, should be measured when harmonics are added in both the voltage and the current. The test can be performed using the quadriform and peaked waveforms specified in Table1 and Fig. 3 respectively. 
Table 1. Electric parameter require values of quadriform

\begin{tabular}{|c|c|c|c|c|}
\hline $\begin{array}{l}\text { Order of } \\
\text { harmonics }\end{array}$ & $\begin{array}{l}\text { Current } \\
\text { phase } \\
\text { amplitude }\end{array}$ & $\begin{array}{l}\text { Current } \\
\text { phase Angle }\end{array}$ & $\begin{array}{l}\text { Voltage } \\
\text { amplitude }\end{array}$ & $\begin{array}{l}\text { Voltage } \\
\text { Angle }\end{array}$ \\
\hline 1 & $100 \%$ & $0^{\circ}$ & $100 \%$ & $0^{\circ}$ \\
\hline 3 & $30 \%$ & $0^{\circ}$ & $3.8 \%$ & $180^{\circ}$ \\
\hline 5 & $18 \%$ & $0^{\circ}$ & $2.4 \%$ & $180^{\circ}$ \\
\hline 7 & $14 \%$ & $0^{\circ}$ & $1.7 \%$ & $180^{\circ}$ \\
\hline 11 & $9 \%$ & $0^{\circ}$ & $1.0 \%$ & $180^{\circ}$ \\
\hline 13 & $5 \%$ & $0^{\circ}$ & $0.8 \%$ & $180^{\circ}$ \\
\hline
\end{tabular}

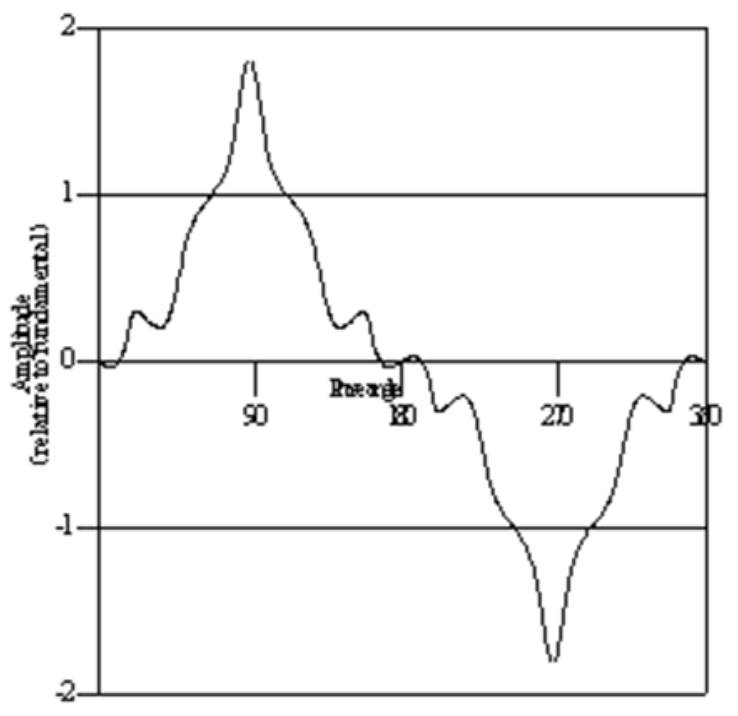

Figure 3. Peaked waveform

The amplitude of a single harmonic should not be higher than $0.12^{U_{1} / h}$ for voltage and $I_{1} / h$ for the current, where $\boldsymbol{h}$ is the harmonic number, and $U_{1}$ and $I_{1}$ are the respective fundamentals. The r. m. s. current may not exceed $I_{\max }$, i.e. for Table 1,the fundamental current component $I_{1}$ may not exceed $0.93^{I_{\max }}$. The peak value of the current may not exceed $1.4^{I_{\max }}$, i.e. for Fig. 3 , the fundamental current component ${ }^{I_{1}}$ (r. m. s.) may not exceed $0.568^{I_{\max }}$. Harmonic amplitudes are calculated relative to the amplitude of the fundamental frequency component of the voltage or current respectively. Phase angle is calculated relative to the zero-crossing of the fundamental frequency voltage or current component respectively.

\subsubsection{Sub-harmonics in the AC current circuit}

The test of sub-harmonics in the AC current circuit is to verify that the error shift due to influence of sub-harmonics in the AC current circuit. The error shift, compared to the intrinsic error at sinusoidal conditions, shall be measured when the sinusoidal reference current is replaced by another sinusoidal signal with twice the peak value, and which is switched on and off every second period as shown by Fig. 4. The measured power should then be the same as for the original sinusoidal signal while the $r$. 
m. s. current is 1.41 times higher. It should be noted that no significant DC current is introduced. During the test, the peak value of the current shall not exceed $1.4^{I_{\max }}$.

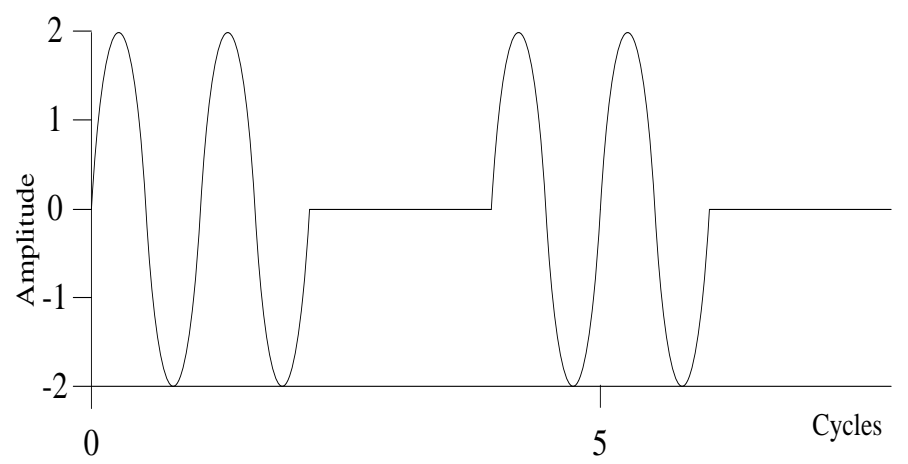

Figure 4. Test waveform of sub-harmonics in the AC current circuit

\subsubsection{Harmonics in the AC current circuit}

The test of harmonics in the AC current circuit is to verify that the error shift due to influence of harmonics in the AC current circuit. The error shift, compared to the intrinsic error at sinusoidal conditions, should be measured when the sinusoidal reference current is replaced by a current with twice the original peak value where the sinusoidal waveform is set to zero during the first and third quarters of the period. The measured power should then be the same as for the original sinusoidal signal while the r. m. s. current is 1.41 times higher. During the test, the peak value of the current should not exceed $1.4^{I_{\max }}$.

\subsubsection{Test results}

The above-mentioned three trials test results are shown in Table 2.

Table 2. Test results

\begin{tabular}{|c|c|c|c|c|c|}
\hline \multirow{2}{*}{ Test item } & Test waveform & \multirow{2}{*}{$\cos \varphi$} & \multirow{2}{*}{ Current value } & \multicolumn{2}{|c|}{ (Error shift value)\% } \\
\cline { 5 - 6 } & & & & First test & Second test \\
\hline $\begin{array}{c}\text { Harmonics in voltage } \\
\text { and current }\end{array}$ & quadriform waveform & 1.0 & $10 \mathrm{Itr}$ & 0.02 & 0.03 \\
\cline { 2 - 6 } & peaked waveform & 1.0 & $10 \mathrm{Itr}$ & 0.05 & 0.07 \\
\hline $\begin{array}{c}\text { Sub-harmonics in the } \\
\text { AC current circuit }\end{array}$ & requirements of 3.3.2 & 1.0 & $10 \mathrm{Itr}$ & 0.03 & 0.05 \\
\hline $\begin{array}{c}\text { harmonics in the AC } \\
\text { current circuit }\end{array}$ & requirements of 3.3.3 & 1.0 & $10 \mathrm{Itr}$ & 0.14 & 0.13 \\
\hline
\end{tabular}

From Table 2, the basic error of electrical energy meter has little influenced under harmonics in voltage and current, sub-harmonics in the AC current circuit and harmonics in the AC current circuit test. The greater influence is the condition of harmonics in the AC current circuit, the maximum error change quantity is $0.14 \%$, nearly equal to $1 / 10$ of the maximum permissible error corresponding to electrical energy meter accuracy level. Harmonics in voltage and current and sub-harmonics in the AC current circuit have no effect on electrical energy meter nearly. 


\section{Conclusion}

As a large number of nonlinear loads in power grid appeared, there are a lot of new requirements for traditional electric measuring instruments, especially the electric energy measuring instruments. The electrical energy meter manufacturers are forced to improve technology to reduce impact of harmonics, such as new energy measuring methods, the computing chips of satisfying the requirement of harmonic measurement, improve the frequency bandwidth of transformer etc. In this paper, the above theoretical analysis and experiment showed that electric energy measuring in influence of harmonics to qualified electric energy meter is little. If the error shift is large under the influence of harmonic and sinusoidal conditions, which illustrates it is appeared quality problem and can't use as measuring instruments of electric energy. This paper studied electrical energy meter influence of measuring energy under the condition of harmonics. The experimental contents and results are very beneficial for the electrical energy meter manufacturer, scientific research workers of using and supervision department.

\section{References}

1. YAO Li. The method of electric energy measurement under the influence of harmonics[J]. Electrical Measurement \& Instrumentation, 42(478): 20-22, (2005).

2. QIU Tao xin. The influence research of energy metering with power harmonic[D]. Guangdong: South China university of Technology master's degree thesis, (2012) .

3. YU Zhi bin, HU Han mei. Measurement and analysis of effect of harmonics on electronic watt-hour meter [J]. China Science and Technology Information, 4(22): 324-325, (2007).

4. YANG Xiao ke, ZHANG Zhong huai. A method of electric energy measurement and quality analysis in power supply system with harmonics [J]. Electrical Measurement \& Instrumentation, , 8: 16-19(2004).

5. 6100B/6105A electrical power standard users manuals. Fluke Corporation (2015). 